\title{
Efecto de la adición de ajo en la estabilidad y calidad sensorial de una pasta de aceituna
}

\author{
Por M. Schwartz ${ }^{\mathrm{a}}$, V. Quitral $^{\mathrm{b}^{*}}$, C. Daccarett ${ }^{\mathrm{a}}$ y R. Callejas ${ }^{\mathrm{a}}$ \\ ${ }^{a}$ Departamento de Agroindustria. Facultad de Ciencias Agronómicas. Universidad de Chile \\ ${ }^{b}$ Departamento de Nutrición. Facultad de Medicina. Universidad de Chile \\ ( ${ }^{*}$ Autor para la correspondencia: vquitral@ med.uchile.cl)
}

\begin{abstract}
RESUMEN
Efecto de la adición de ajo en la estabilidad y calidad sensorial de una pasta de aceituna

Se evaluó el efecto de ajo en la calidad y estabilidad de una pasta de aceitunas. Se preparó una pasta control $(\mathrm{Pc})$ a base de aceitunas variedad Sevillana y otra idéntica con adición de ajo $(\mathrm{Pa})$ en concentración de $0.5 \mathrm{~g} / 100 \mathrm{~g}$. Se realizaron análisis microbiológicos, fisicoquímicos y sensoriales en ambas pastas al tiempo cero y durante el almacenamiento hasta los 90 días a $4^{\circ} \mathrm{C}$. Los parámetros microbiológicos permanecen dentro de los límites aceptados desde el punto de vista sanitario. La adición de ajo a la pasta de aceitunas produce aumento de acidez, expresada como \% ácido láctico, y disminución significativa ( $p$ $<0.05)$ de $\mathrm{pH}$ a los 30 y 60 días de almacenamiento. La adición de ajo produce menor aumento del índice de peróxido a los 90 días de almacenamiento. El análisis instrumental de color indica que Pa es más luminoso y más intenso. La aceptabilidad es similar entre las pastas. La calidad sensorial indica que $\mathrm{Pa}$ tiene mejor apariencia, aroma, consistencia y sabor. La rancidez es menos percibida en $\mathrm{Pa}$ que en $\mathrm{Pc}$, siendo en ambas bajo. El gusto ácido, salado y amargo es menor en $\mathrm{Pa}$. De acuerdo a los resultados, la adición de ajo es recomendable para pasta de aceitunas.
\end{abstract}

PALABRAS CLAVE: Aceituna - Acidez - Ajo - Análisis sensorial - Color - Índice de peróxido - $\mathrm{pH}$.

\section{SUMMARY}

Effect of the addition of garlic on the stability and sensory quality of olive paste

The effect of garlic on the quality and stability of olive paste was evaluated. A control paste (Pc) was prepared based on a Sevillana olive variety, the other was identical to $\mathrm{Pc}$ with $0.5 \mathrm{~g} / 100 \mathrm{~g}$ of garlic $(\mathrm{Pa})$. Microbiological, physicochemical and sensory changes were monitored in both pastes at time zero and during storage up to 90 days at $4^{\circ} \mathrm{C}$. Microbiological parameters remain within acceptable limits from the point of view of health. The addition of garlic to the olive paste leads to increased acidity (\% lactic acid) and a significant decrease $(\mathrm{p}<0.05)$ in $\mathrm{pH}$ at 30 and 60 days of storage. The addition of garlic produces a lower increase in peroxide value after 90 days of storage. An instrumental color analysis indicates that $\mathrm{Pa}$ is brighter and has greater color intensity. Acceptability is similar between the pastes. The sensory quality indicates that $\mathrm{Pa}$ has better appearance, aroma, texture and flavor.
Rancidity is less than perceived in $\mathrm{Pa}$. The tastes sour, salty and bitter are lower in $\mathrm{Pa}$. According to the results, the addition of garlic is recommended for olive paste and similar foods.

KEY-WORDS: Acidity - Color - Garlic - Olives Peroxide value $-\mathrm{pH}-$ Sensory analysis.

\section{INTRODUCCIÓN}

El ajo es una planta del género Allium que tiene un bulbo que se usa para dar sabor a los alimentos, históricamente ha sido utilizado como planta medicinal para diferentes tipos de afecciones. Estudios epidemiológicos y clínicos sugieren que el ajo está relacionado con el riesgo de enfermedades cardiacas y cáncer (Thomson et al., 2006; Ngo et al., 2007; Omar \& Al-Wabel, 2010); ha sido asociado con la disminución de la presión sanguínea de significancia clínica en pacientes con hipertensión (Ried et al., 2010). Fleischauer et al. (2000) publicaron un meta-análisis que indica que el alto consumo de ajo está relacionado con la disminución del riesgo de cáncer al estómago y colorrectal.

El ajo mejora la estabilidad de los alimentos ya que contiene muchos compuestos bioactivos como polifenoles y organosulfurados (Queiroz et al., 2009); posee actividad antimicrobiana (Hughes et al., 1991; Fujisawa et al., 2008; Tajkarimi et al., 2010) y antioxidante en alimentos (Aguirrezábal et al., 2000; Borek, 2001; Şener et al., 2007; Bozin et al., 2008; Janoszka, 2010)

Las aceitunas de la variedad Sevillana se destinan principalmente al mercado de aceitunas de mesa; del total de producción destinada a la comercialización, alrededor del 10 al $15 \%$ de los frutos se rechazan en la industria, por presentar manchas, golpes o estar ralladas. Estas "aceitunas de rechazo" se pueden utilizar en la elaboración de pasta untable, como una alternativa saludable frente a otros alimentos como margarinas, paté y otras pastas de alto valor calórico. (Callejas et al., 2001; Schwartz et al., 2009) 
El objetivo del presente trabajo es evaluar el efecto de ajo en la calidad y estabilidad de una pasta de aceitunas.

\section{MATERIALES Y MÉTODOS}

\subsection{Materia prima}

Se utilizó ajo fresco, el que fue desinfectado (con una solución de hipoclorito de sodio $\mathrm{NaClO}$ al $0.05 \%$ ) y triturado.

Se elaboró una pasta untable de aceitunas variedad Sevillana de acuerdo a Schwartz et al. (2009). Para obtener la pulpa de aceitunas, éstas se deshuesaron y trituraron, se agregaron preservantes (sorbato de potasio y benzoato de sodio en $2 \mathrm{~g} / \mathrm{kg}$ y $1 \mathrm{~g} / \mathrm{kg}$ respectivamente), se ajustó el $\mathrm{pH}$ a valores de $4.1-4.4$ con ácido láctico, se envasaron y almacenaron a $4^{\circ} \mathrm{C}$. Se preparó una pasta control $(\mathrm{Pc})$ y una pasta con adición de ajo $(\mathrm{Pa})$ en una concentración de $0.5 \mathrm{~g} / 100 \mathrm{~g}$. Las pastas se evaluaron durante el tiempo de almacenamiento.

Se analizaron 3 muestras de cada tipo de pasta realizando los análisis por duplicado en cada punto de análisis.

\subsection{Análisis}

\section{Análisis microbiológicos}

Se realizó recuento de microorganismos aerobios mesófilos viables (R.A.M.) (Norma Chilena NCh2659of2002); recuento de enterobacterias (Norma Chilena NCh2676.of2002); recuento de hongos y levaduras (Norma Chilena NCh 2734.Of2002) a las muestras a los 0, 30, 60 y 90 días de almacenamiento.

\section{Análisis fisicoquímicos}

Se aplicaron las metodologías descritas en A.O.A.C. (1995) para la determinación de $\mathrm{pH}$ y acidez titulable. La estabilidad de la materia grasa extraída de la pasta de aceitunas se evaluó mediante el índice de peróxido (A.O.C.S., 1993). El color se analizó instrumentalmente con un colorímetro Minolta CR-200b evaluando los parámetros $L^{*}, a^{*}, b^{*}$ y calculando saturación de color $\mathrm{C}^{*}\left[\left(\mathrm{a}^{2}+\mathrm{b}^{2}\right)^{0.5}\right]$ (Hand et al., 1953). La actividad de agua (Aw) se determinó con un analizador LUFFT modelo 5803.

\section{Análisis sensorial}

Se evaluó la aceptabilidad con un panel no entrenado mediante una escala no estructurada de 15 puntos, que indica "me disgusta extremadamente" con valor 0 hasta "me gusta extremadamente" con valor 15. La calidad se evaluó con panel entrenado de 15 personas con escala no estructurada de 15 puntos, evaluando apariencia, color, aroma, rancidez, gusto ácido, salado, amargor, consistencia y sabor. (Wittig, 2001; AnzaldúaMorales, 1994)

\section{Análisis estadístico}

Los resultados se analizaron con Análisis de Varianza (ANOVA) y prueba de rango múltiple de Duncan. Se utilizó el Programa SPSS 11.5.

\section{RESULTADOS Y DISCUSIÓN}

\subsection{Análisis microbiológicos}

El recuento total de bacterias aerobias mesófilas se presenta en la Tabla 1. Se observa que solo a los 90 días $\mathrm{Pa}$ presenta menor recuento, sin ser significativo. El recuento de enterobacterias y hongos y levaduras fue $<10$ (ufc/ml) en $\mathrm{Pc}$ y $\mathrm{Pa}$ durante todo el tiempo de almacenamiento.

Ambas pastas presentan recuento menor que el exigido en el Reglamento Sanitario de los Alimentos de Chile (Ministerio de Salud, 2010) para "alimentos listos para el consumo"; por lo tanto se pueden consumir hasta los 90 días.

Existen dos etapas durante la elaboración de las pastas de aceitunas que inhiben el desarrollo microbiano, una de ellas es la adición de sorbato de potasio $(2 \mathrm{~g} / \mathrm{kg})$ y benzoato de sodio $(1 \mathrm{~g} / \mathrm{kg})$ a la pulpa después de haber deshuesado y triturado. Estos preservantes químicos tienen reconocido efecto sobre hongos, levaduras y bacterias (Miyhar et al., 1997; Guynot et al., 2005; Arroyo-López et al., 2008; Hracek et al., 2010).

Otra etapa que se realiza durante la elaboración de la pasta de aceitunas es el ajuste de $\mathrm{pH}$ a valores de 4.1 - 4.4 con ácido láctico. Los alimentos listos para el consumo, como la pasta de aceituna, con valores de $\mathrm{pH}$ menores de 4.4 se considera que no favorecen el desarrollo de Listeria monocytogenes (Ministerio de Salud, 2010), microorganismo emergente de gran preocupación en la actualidad.

Los estudios que han comprobado el efecto del $\mathrm{pH}$ y tipo de ácido en la inactivación de microorganismos proponen dos mecanismos inhibitorios: (i) acidificación intracelular con pérdida de homestasis y (ii) efecto específico de la forma no disociada del ácido sobre actividad metabólica. (Miller et al., 2009)

Tabla 1

Recuento total de bacterias aerobias mesofilas (ufc/ml) durante el tiempo de almacenamiento

\begin{tabular}{ccccc}
\hline \multirow{2}{*}{ Muestra } & \multicolumn{4}{c}{ Tiempo de almacenamiento (días) } \\
\cline { 2 - 5 } & $\mathbf{0}$ & $\mathbf{3 0}$ & $\mathbf{6 0}$ & $\mathbf{9 0}$ \\
\hline $\mathrm{PC}$ & $<10$ & $<10$ & $<10$ & $5.7 \times 10^{1}$ \\
$\mathrm{~Pa}$ & $<10$ & $<10$ & $<10$ & $5.4 \times 10^{1}$ \\
\hline
\end{tabular}


Otro aspecto que hay que considerar es que las aceitunas contienen polifenoles, con efecto inhibitorio sobre microorganismos (Arroyo-López et al., 2005; Papadopoulou et al., 2005). Diversos autores han reportado que hidroxitirosol, que es el compuesto fenólico de mayor concentración en aceitunas de mesa (Boskou et al., 2006; Malheiro et al., 2011) inhibe la tasa de crecimiento de un amplio espectro de bacterias y hongos (Soni et al., 2006).

\subsection{Análisis fisicoquímicos}

La Tabla 2 presenta la variación de pH entre las muestras y durante el tiempo de almacenamiento. En ambas muestras el $\mathrm{pH}$ disminuye a los 90 días de almacenamiento respecto al tiempo 0 . El ajo adicionado a la pasta de aceitunas tiene un efecto acidificante ya que disminuye el $\mathrm{pH}$ respecto a $\mathrm{Pc}$, lo que se confirma con los datos de la Tabla 3 en que existe un aumento significativo de la acidez expresada como \% de acido láctico. Este efecto contribuye a aumentar la estabilidad de la pasta de aceituna.

Yin \& Cheng (2003) no encontraron efecto en el $\mathrm{pH}$ de muestras de carne molida al adicionar compuestos organosulfurados derivados de ajo.

El ajo es rico en $\gamma$-glutamilcisteína y otros compuestos azufrados, también contiene aceite esencial, flavonoides, antocianinas, lectinas, fructanos, pectinas, vitaminas $B_{1}, B_{2}, B_{6}, C$ y $E$, biotina, ácido nicotínico, ácidos grasos, glicolípidos, fosfolipidos y aminoácidos esenciales (Bozin et al., 2008; Şener et al., 2007); los que en su conjunto tienen la capacidad de disminuir el $\mathrm{pH}$ de la pasta de aceitunas.
Tabla 2

Variación de pH en las muestras durante el tiempo de almacenamiento

\begin{tabular}{ccccc}
\hline \multirow{2}{*}{ Muestra } & \multicolumn{4}{c}{ Tiempo de almacenamiento (días) } \\
\cline { 2 - 5 } & $\mathbf{0}$ & $\mathbf{3 0}$ & $\mathbf{6 0}$ & $\mathbf{9 0}$ \\
\hline $\mathrm{PC}$ & $4.30^{\mathrm{bA}}$ & $4.39^{\mathrm{aA}}$ & $4.16^{\mathrm{CA}}$ & $4.17^{\mathrm{CA}}$ \\
$\mathrm{Pa}$ & $4.10^{\mathrm{bA}}$ & $4.15^{\mathrm{aB}}$ & $3.93^{\mathrm{CB}}$ & $3.96^{\mathrm{CA}}$ \\
\hline
\end{tabular}

Letras minúsculas diferentes indican diferencia significativa en la fila $(p<0.05)$. Letras mayúsculas diferentes indican diferencias significativas en columna $(p<0.05)$.

Tabla 3

Variación de acidez (\%Ac. Láctico) en las muestras durante el tiempo de almacenamiento

\begin{tabular}{ccccc}
\hline \multirow{2}{*}{ Muestra } & \multicolumn{4}{c}{ Tiempo de almacenamiento (días) } \\
\cline { 2 - 5 } & 0 & 30 & 60 & 90 \\
\hline $\mathrm{PC}$ & $0.43^{\mathrm{aA}}$ & $0.41^{\mathrm{aA}}$ & $0.41^{\mathrm{aA}}$ & $0.43^{\mathrm{aA}}$ \\
$\mathrm{Pa}$ & $0.53^{\mathrm{aB}}$ & $0.54^{\mathrm{abB}}$ & $0.57^{\mathrm{bB}}$ & $0.57^{\mathrm{bB}}$ \\
\hline
\end{tabular}

Letras minúsculas diferentes indican diferencia significativa en la fila $(p<0.05)$. Letras mayúsculas diferentes indican diferencias significativas en columna $(p<0.05)$.

El índice de peróxido aumenta significativamente $(p<0.05)$ durante el tiempo en ambas muestras, pero en $\mathrm{Pa}$ tiende a estabilizarse a los 60 y 90 días, mientras que Pc continúa aumentando (Figura 1).

Las aceitunas que son la materia prima para las pastas, contienen tocoferol, carotenos y polifenoles con efecto antioxidante (Pereira et al., 2006; Anni-

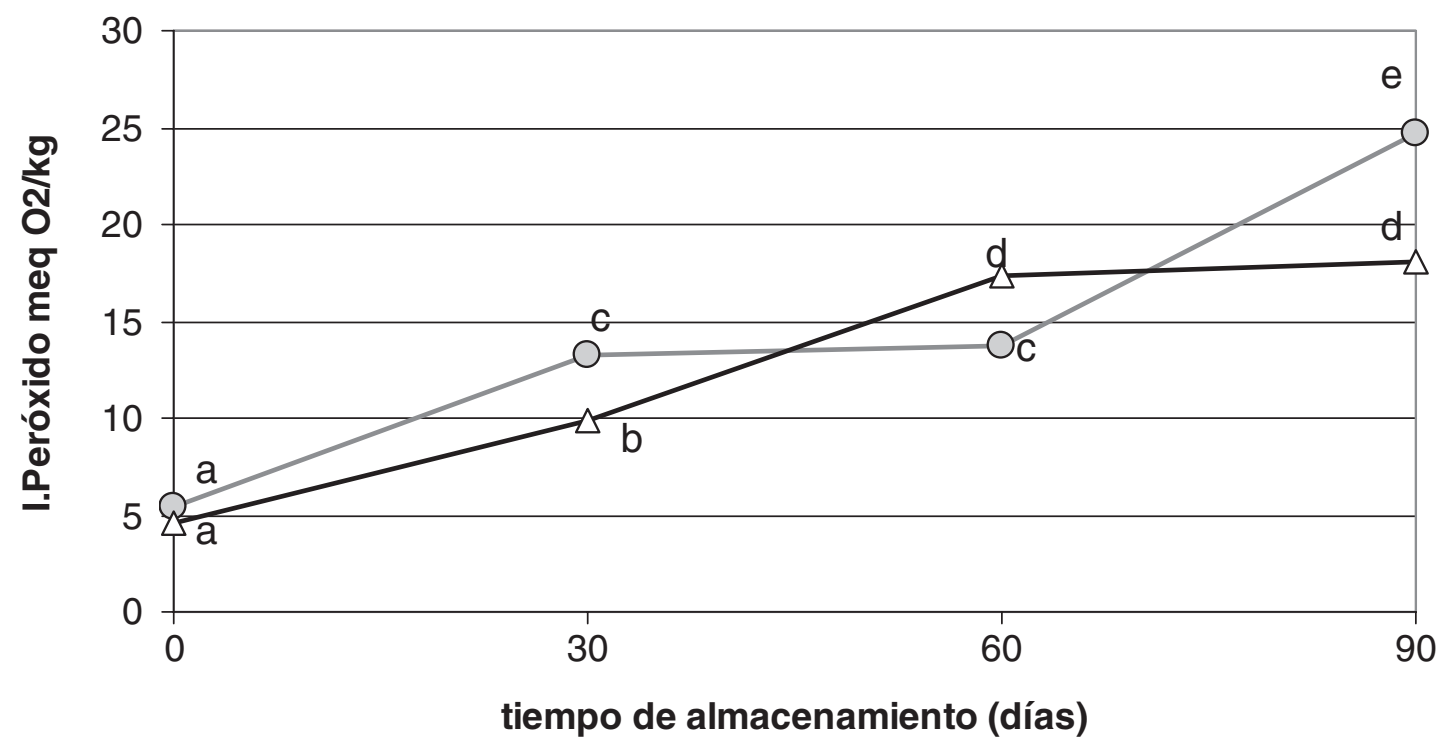

$$
-\mathrm{O}-\mathrm{Pc} \triangle \mathrm{Pa}
$$

Figura 1

Variación del Índice de Peróxido en muestras de pasta de aceitunas durante el tiempo de almacenamiento. Letras distintas indican diferencia significativa con error de $5 \%$. 
va \& Tsimidou, 2009; Rodríguez et al., 2009) y sumados a componentes del ajo, se logra disminuir la oxidación a través del tiempo.

Se han informado valores de polifenoles totales en ajo de $59.4 \mathrm{mg}$ ácido gálico equivalente/100g (Brat et al., 2006) y 92.0 (USDA, 2007). Se considera la alicina el principal responsable de la actividad antioxidante del bulbo de ajo. (Pokorny et al., 2005).

La alicina es un compuesto organosulfurado biológicamente activo que no está presente en la planta intacta, es formada cuando se cortan o trituran los bulbos de ajo debido a una reacción enzimática entre aminoácidos no proteicos y la enzima aliinasa. Los efectos de la alicina se atribuyen a su grupo tiol. (Cavagnaro et al., 2007)

Existen estudios que han comprobado el efecto antioxidante de los compuestos organosulfurados contra la oxidación lipídica (Banerjee et al., 2003; Tapiero et al., 2004; Liu et al., 2005; Şener et al., 2007). Este fenómeno se debe a la activación y modificación de diferentes enzimas y a mecanismos reductores no enzimáticos y capacidad de quelación de cationes metálicos. Diail sulfuro y dialil disulfuro, son los compuestos organosulfurados lipofílicos que proveen protección antioxidante y antimicrobiana (Yin y Cheng, 2003).

Mediante el índice de peroxido Sallam et al. (2004) demostraron efecto antioxidante de ajo fresco en alimentos, mientras que Navas et al (2006) lo demostraron en aceite al aplicar extracto de ajo. Papadopoulou \& Roussis (2008) comprobaron la capacidad antioxidante de ajo en aceite de maíz durante el almacenamiento, cocción y fritura.

La actividad de agua (Aw) se mantiene entre 0.90 y 0.92 , sin diferencias significativas durante el almacenamiento ni entre las muestras. Este valor de Aw permite que en el alimento no se favorezca el desarrollo de Listeria monocytogenes. (Ministerio de Salud, 2010).

En cuanto al análisis de color podemos observar de la Tabla 4 que el parámetro $L^{*}$, que representa la luminosidad, es mayor en la muestra $\mathrm{Pa}$, es decir el ajo le aporta más claridad a la pasta. La disminución de $L^{*}$ al final del almacenamiento, para ambas muestras, puede ser causa de la oxidación de los polifenoles (García et al., 1999). El valor L* se ha usado como indicador del pardeamiento en vegetales (Quevedo et al., 2009) y se demuestra
Tabla 4

Variación de parámetro L* (luminosidad) en las muestras durante el tiempo de almacenamiento

\begin{tabular}{ccccc}
\hline \multirow{2}{*}{ Muestra } & \multicolumn{4}{c}{ Tiempo de almacenamiento (días) } \\
\cline { 2 - 5 } & $\mathbf{0}$ & $\mathbf{3 0}$ & $\mathbf{6 0}$ & $\mathbf{9 0}$ \\
\hline $\mathrm{PC}$ & $51.37^{\mathrm{bA}}$ & $50.07^{\mathrm{aA}}$ & $51.17^{\mathrm{bA}}$ & $49.43^{\mathrm{aA}}$ \\
$\mathrm{Pa}$ & $52.43^{\mathrm{bA}}$ & $52.28^{\mathrm{abB}}$ & $52.95^{\mathrm{bB}}$ & $50.67^{\mathrm{aA}}$ \\
\hline
\end{tabular}

Letras minúsculas diferentes indican diferencia significativa en la fila $(p<0.05)$. Letras mayúsculas diferentes indican diferencias significativas en columna $(p<0.05)$.

que es menor en $\mathrm{Pa}$, gracias a su efecto antioxidante.

Los valores determinados en las muestras de pasta de aceitunas son menores a los de aceitunas de mesa determinados por Arroyo-López et al. (2005) y Romeo et al. (2009)

Los cambios de cromaticidad se pueden deber a la degradación de la clorofila, al pardeamiento y polimerización de polifenoles. (Minguez-Mosquera et al., 1989; Arroyo-López et al., 2008; SegoviaBravo et al., 2009)

Los valores de $b^{*}$ en las muestras de pasta de aceitunas son menores que 34.8, determinados en aceitunas de mesa por Arroyo López et al. (2005) y que 35.6 determinado en pulpa de aceitunas por Romeo et al. (2009)

De acuerdo a la Tabla 6, la diferencia de color entre 0 y 60 días es baja en ambas muestras, aumentando la diferencia entre 60 y 90 días, pero los valores son menores de 2.8 CIELAB, de acuerdo a Lozano (1978) y Melgosa et al. (2001) con valores entre 1.1 y 2.8 puede hablarse de una tolerancia de color "rigurosa".

\subsection{Análisis sensorial}

La aceptabilidad se encuentra entre los valores 9 a 11, que en la escala no estructurada de $15 \mathrm{~cm}$ indica que las muestras de pasta de aceituna les gustan a los consumidores. Pa tiene mayor puntuación que Pc al tiempo 0 y los 90 días ocurre el efecto contrario. No existen diferencias significativas entre las muestras ni durante el tiempo.

La calidad evaluada en escala no estructurada para distintos parámetros no presenta diferencias

Tabla 5

Variación de parámetro $a^{*}$ y b* en muestras de pasta de aceitunas durante el tiempo de almacenamiento

\begin{tabular}{|c|c|c|c|c|c|c|c|c|}
\hline \multirow{3}{*}{ Muestra } & \multicolumn{8}{|c|}{ Tiempo de almacenamiento (días) } \\
\hline & \multicolumn{2}{|c|}{0} & \multicolumn{2}{|c|}{30} & \multicolumn{2}{|c|}{60} & \multicolumn{2}{|c|}{90} \\
\hline & $\mathbf{a}^{*}$ & $\mathbf{b}^{*}$ & $\mathbf{a}^{*}$ & $\mathbf{b}^{*}$ & $a^{*}$ & $\mathbf{b}^{*}$ & $\mathbf{a}^{*}$ & $\mathbf{b}^{*}$ \\
\hline $\mathrm{Pc}$ & -1.35 & 23.15 & -1.37 & 23.10 & -0.95 & 23.90 & -1.32 & 22.60 \\
\hline $\mathrm{Pa}$ & -1.65 & 24.72 & -1.55 & 24.38 & -0.92 & 24.37 & -0.78 & 22.80 \\
\hline
\end{tabular}


Tabla 6

Diferencia de color en muestras de pasta de aceituna durante el tiempo de almacenamiento

\begin{tabular}{ccc}
\hline \multirow{2}{*}{$\begin{array}{c}\text { Diferencia de tiempos } \\
\text { de almacenamiento }\end{array}$} & \multicolumn{2}{c}{ Diferencia de color $\left(\Delta \mathrm{E}^{*} \mathrm{ab}\right)$} \\
\cline { 2 - 3 } & $\mathbf{P c}$ & $\mathbf{P a}$ \\
\hline $30-0$ & 1,30 & 0,38 \\
$60-30$ & 1,42 & 0,92 \\
$90-60$ & 2,20 & 2,77 \\
\hline
\end{tabular}

significativas entre el tiempo 0 y 90 días de almacenamiento. La figura 2 presenta la evaluación realizada a los 90 días de almacenamiento para los distintos parámetros considerados. La apariencia es más compacta que disgregada y la consistencia es más untable que granulosa en ambas muestras, lo que demuestra que cumple con el requisito de "pasta" sin producirse separación de componentes ni granulosidad. Ambos parámetros son mejor evaluados en $\mathrm{Pa}$ que en Pc.

De acuerdo a la figura 2 los valores de color se encuentran cercanos al punto central de la escala no estructurada, por lo tanto el color es calificado en la mitad de "muy pálido" y "muy intenso", siendo Pa más pálido, lo que concuerda con los valores instrumentales de luminosidad $L^{*}$ y cromaticidad $a^{*}$ y b*. Gibis (2007) aplicó ajo a marinadas con aceite de oliva para freir hamburguesas y el aumento de la concentración de ajo (de 2 a 20 g/100g) provocó oscurecimiento.

El aroma se evaluó entre "sin aroma" y "muy aromático", la muestra Pa presenta mayor aroma que $\mathrm{Pa}$, siendo ambos suaves, lo que para una pasta es adecuado ya que puede acompañar a diferentes tipos de alimentos sin provocar saturación en los consumidores. Un estudio realizado por Gibis (2007) indica que concentraciones de $20 \mathrm{~g} / 100 \mathrm{~g}$ de ajo en marinadas provocó menor aceptación para olor que muestras con $2 \mathrm{~g} / 100 \mathrm{~g}$. En el presente estudio la concentración de ajo es de $0.5 \mathrm{~g} / 100 \mathrm{~g}$, ya que mayores concentraciones producen aromas muy fuertes que no son del agrado del consumidor, por compuestos organosulfurados volátiles como alicina (Fujisawa et al., 2008)

Los consumidores perciben una rancidez muy leve y es menor para $\mathrm{Pa}$, lo que concuerda con el análisis químico de índice de peróxido.

El gusto ácido, salado y amargor se encuentran cercanos al punto medio de la escala no estructurada de evaluación, los que son adecuados a un producto a base de aceitunas. El sabor fue evaluado mas cercano a "muy característico" Pa obtiene mayor puntuación, por lo que el ajo realza el sabor característico de las aceitunas.

\section{CONCLUSIONES}

La adición de ajo $(0.5 \mathrm{~g} / 100 \mathrm{~g})$ a pasta de aceitunas permite aumentar su estabilidad ya que aumenta la acidez y disminuye el índice de peróxido durante el almacenamiento. La calidad sensorial es levemente superior en la pasta con ajo.

\section{AGRADECIMIENTOS}

Esta investigación se realizó gracias al financiamiento de CORFO del Gobierno de Chile.

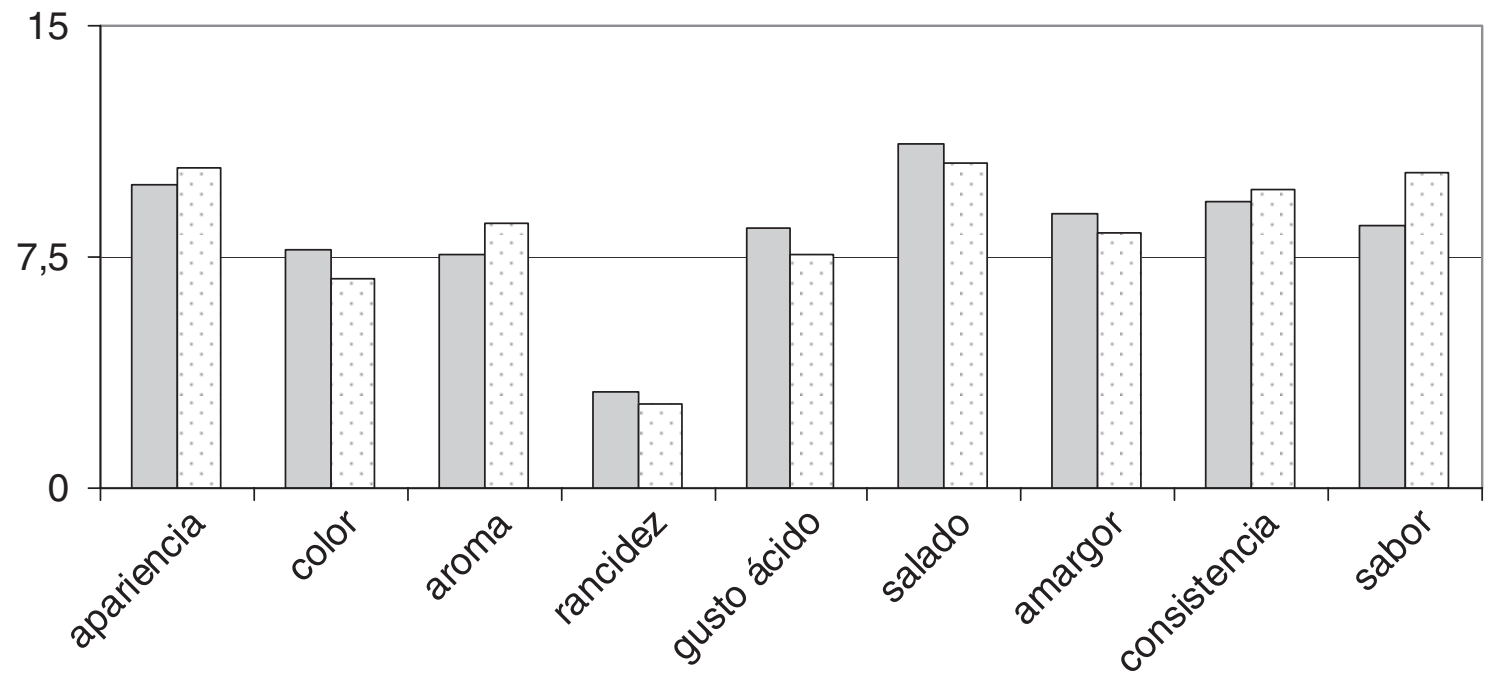

\section{$\square \mathrm{Pc} \quad \square \mathrm{Pa}$}




\section{REFERENCIAS}

Aguirrezábal MM, Mateo J, Domínguez MC, Zumalacárregui JM. 2000. The effect of paprika, garlic and salt on rancidity in dry sausages. Meat Sci. 54, 77-81.

A.O.A.C, 1995. Official Methods of Análysis of the Association of Official Analytical Chemist International. Volumes I y II. $16^{\text {th }}$ edition. Maryland, U.S.A.

A.O.C.S. 1993. Oficial Methods and Recommended Practices of the American Oil Chemists'Society. $3^{\text {th }}$ Edition. Champaign, Illinois.

Anniva C, Tsimidou MZ. 2009. On the quality control of "olive paste", a specialty base on olives and olive oil. Eur. J. Lipid Sci. Technol. 111, 328-336.

Anzaldúa-Morales A. 1994. La evaluación sensorial de los alimentos en la teoría y la práctica. Editorial Acribia, España.

Arroyo-López FN, Bautista-Gallego J, Durán-Quintana MC, Garrido-Fernandez A. 2008. Effects of ascorbic acid, sodium metabisulfite and sodium chloride on freshness retention and microbial growth during the storage of Manzanilla-Aloreña cracked table olives. LWT - Food Sci. Tech. 41, 551-560.

Arroyo-López FN, Bautista-Gallego J, Segovia-Bravo KA, García-García P, Durán-Quintana MC, Romero C, Rodríguez-Gómez F, Garrido-Fernández A. 2009. Instability profile of fresh packed "seasoned" Manzanilla-Aloreña table olives. LWT - Food Sci. Technol. 42, 1629-1639.

Arroyo López FN, Romero C, Duran MC, López A, García P, Garrido A. 2005. Kinetic study of the physicochemical and microbiological changes in "seasoned" olives during the shelf-life period. J. Agric. Food Chem. 53, 5285-5292.

Banerjee S, Sood S, Dinda AK, Das T, Maulik SK. 2003. Chronic oral administration of raw garlic protects against isoproterenol-induced myocardial necrosis in rat. Comp. Biochem. Physiol. Part C. 136, 377-386.

Borek C. 2001. Antioxidant health effects of aged garlic extract. J. Nutr. 131, 1010S-1015S

Boskou G, Salta FN, Chrysostomou S, Mylona A, Chiou A, Andrikopoulo NK. 2006. Antioxidant capacity and phenolic profile of table olives from the Greek market. Food Chem. 94, 558-564.

Bozin B, Mimica-Dukic N, Samojlik I, Goran A, Igic R. 2008. Phenolics as antioxidants in garlic (Allium sativum L., Alliaceae). Food Chem. 111, 925-929.

Brat $P$, Georgè S, Bellamy A, Du Chaffaut L, Scalbert A, Mennen L, Arnault N, Amiot MJ. 2006. Daily polyphenol intake in France from fruit and vegetables. J. Nutr. 136, 2368-2373.

Callejas R, Barraza J, Kania A. 2001. Evaluación durante la cosecha de los principales factores que provocan el rechazo de aceitunas. J. Olivícolas Nacionalles, 79-81

Cavagnaro PF, Camargo A, Galmarini CR, Simon Philipp W. 2007. Effect of cooking on garlic (Allium sativum L.) antiplatalet activity and thiosulfinates content. $J$. Agric. Food Chem. 55, 1280-1288.

Fleischauer AT, Poole C, Arab L. 2000. Garlic consumption and cancer prevention: meta-analyses of colorectal and stomach cancers. Am. J. Cli. Nutr. 72, 1047-1052.

Fujisawa H, Suma K, Origuchi K, Kumagai H, Seki T, Ariga T. 2008. Biological and chemical stability of garlicderived allicin. J. Agric. Food Chem. 56, 4229-4235.

García P, Brenes M, Romero C, Garrido A. 1999. Color and texture of acidified ripe olives in pouches. J. Food Sci. 64, 248-251.
Gibis M. 2007. Effect of oil marinades with garlic, onion and lemon juice on the formation of heterocyclic aromatic amines in fried beef patties. J. Agric. Food Chem. 55, 10240-10247.

Guynot ME, Ramos AJ, Sanchis V, Marín S. 2005. Study of benzoate, propionate, and sorbate salts as mould spoilage inhibitors on intermediate moisture bakery products of low pH (4.5-5.5). Intl. J. Food Microbiol. 101, 161-168.

Hand DB, Robinson WB, Wishnetsky T, Ransford JR. 1953. Color measurement: Application to food quality grades. J. Agric. Food Chem. 1, 1209-1212.

Hracek VM, Gliemmo MF, Campos CA. 2010. Effect of steviosides and system composition on stability and antimicrobial action of sorbates in acidified model aqueous systems. Food Res. Int. 43, 2171-2175.

Hughes BG Lawson LD. 1991. Antimicrobial effects of Allium sativum (garlic), Allium ampeloprasum (elephant garlic) and Allium cepa (onion), garlic compounds and commercial garlic supplement products. Phytother. Res. 5, 154-158.

Janoszka B. 2010. 7-Ketocholesterol and 7-hydroxycholesterol in pork meat and its gravy thermally treated without additives and in the presence of onion and garlic. Meat Sci. 86, 976-984.

Liu C-T, Hse H, Lii C-K, Chen P-S, Sheen L-Y. 2005. Effects of garlic oil and diallyl trisulfide on glycemic control in diabetic rats. Eur. J. Pharm. 516, 165-173.

Lozano RD. 1978. El color y su medición. Editorial Americalee. Buenos Aires. Argentina

Malheiro RA, Casal S, Bento A, Pereira JA. 2011. Cultivar effect on the phenolic composition and antioxidant potential of stoned table olives. Food Chem Toxicol, 49, 450-457.

Melgosa M, Pérez MM, Yebra A, Huertas R, Hita E. 2001. Algunas reflexiones y recientes recomendaciones internacionales sobre evaluación de diferencias de color. Optica Pura y Aplicada. 34, 1-10.

Mihyar GF, Yamani MI, Al-Sa'ed AK. 1997. Resistance of Yeast Flora of Labaneh to Potassium Sorbate and Sodium Benzoate. J. Dairy Sci. 80, 2304-2309.

Miller FA, Ramos B, Gil MM, Brandão TRS, Teixeira P, Silva C. 2009. Influence of $\mathrm{pH}$, type of acid and recovery media on the thermal inactivation of Listeria innocua. Int J. Food Microbiol. 133, 121-128.

Minguez-Mosquera MI, Garrido-Fernandez J, Gandul-Rojas B. 1989. Pigments changes in olives during fermentation and brine storage. J. Agric. Food Chem. 37, 8-11.

Ministerio de Salud. 2010. Reglamento Sanitario de los Alimentos. DTO. N. 977/96. Actualizado 2010. Santiago. Chile.

Navas PB, Carrasqueño-Durán A, Flores I. 2006. Effect of black tea, garlic and onion on corn oil stability and fatty acid composition Ander accelerated oxidation. Int. J. Food Sci. Technol. 41, 243-247.

NCh 2734.Of2002. Determinación de hongos y levaduras - Técnica de recuento en placa. Instituto Nacional de Normalización. Santiago, Chile.

NCh 2659.of2002. Determinación de microorganismos aerobios mesófilos - Técnica de recuento en placa a $35^{\circ} \mathrm{C}$. Instituto Nacional de Normalización. Santiago, Chile.

NCh 2676.of2002 . Determinación de Enterobacteriaceae sin resucitación - Técnica NMP y técnica de recuento en placa. Instituto Nacional de Normalización. Santiago, Chile.

Ngo SNT, Williams DB, Cobiac L, Head RJ. 2007. Does garlic reduce risk of colorectal cancer? A systematic review. J. Nutr. 137, 2264-2269. 
Omar SH, Al-Wabel NA. 2010. Organosulfur compounds and possible mechanism of garlic in cancer. Saudi Pharm. J. 18, 51-58.

Papadopoulou C, Soulti K, Roussis IG. 2005. Potential antimicrobial activity of red and white wine phenolic extracts against strains of Staphilococcus aureus, Escherichia coli and Candida albicans. Food Tech. Biotech. 43, 41-46.

Papadopoulou D, Roussis IG. 2008. Inhibition of corn oil oxidation by $\mathrm{N}$-acetyl-cysteine and glutathione. Food Chem. 109, 624-629.

Pereira JA, Pereira APG, Ferreira I, Valentão P, Andrade P, Seabra R, Estevinho L, Bento A. 2006. Table olives from Portugal: Phenolic compounds, antioxidant potential and antimicrobial activity. J. Agric. Food Chem. 54, 8425-8431.

Pokorny J, Yanishlieva N, Gordon M. 2005. Antioxidantes de los Alimentos. Editorial Acribia, S.A. Zaragoza. España.

Queiroz YS, Ishimoto EY, Bastos D, Torres E. 2009. Garlic (Allium sativum L.) and ready-to-eat garlic products: In vitro antioxidant activity. Food Chem. 115, 371-374.

Quevedo R, Díaz O, Caqueo A, Ronceros B, Aguilera JM. 2009. Quantification of enzymatic browning kinetics in pear slices using non-homogenous $L^{*}$ color information from digital images. LWT - Food Sci. Tech. 42, 1367-1373.

Ried K, Frank OR, Stocks NP. 2010. Aged garlic extract lowers blood pressure in patients with treated but uncontrolled hypertension: a randomised controlled trial. Maturitas. 67, 144-150.

Rodríguez G, Lama A, Jaramillo S, Fuentes-Alventosa JM, Guillén $R$, Jiménez-Arujo $A$, Rodríguez-Arcos $R$, Fernandez-Bolaños J. 2009. 3,4-Dihydroxyphenylglycol (DHPG): An important phenolic compound present in natural table olives. J. Agric. Food Chem. 57, 6298-6304.

Romeo FV, De Luca S, Piscopo A, Perri E, Poiana M. 2009. Effects of post-fermentation processing on the stabilisation of naturally fermented green table olives (cv Nocellara etnea). Food Chem. 116, 873-878.
Sallam KI, Ishioroshi M, Samejima K. 2004. Antioxidant and antimicrobial effects of garlic in chicken sausage. Lebensm Wiss Technol. 37, 849-855.

Schwartz M, Quitral V, Daccarett C, Callejas J. 2009. Desarrollo de pasta untable de aceituna variedad Sevillana. Grasas y Aceites. 60, 451-457.

Segovia-Bravo K, Jarén-Galán $M$, García-García $P$, Garrido-Fernandez A. 2009. Browning reactions in olives: Mechanism and polyphenols involved. Food Chem. 114, 1380-1385.

Şener G, Sakarcan A, Yeğn BÇ. 2007. Role of garlic in the prevention of ischemia-reperfusion injury. Mol. Nutr. Food Res. 51, 1345-1352.

Soni MG, Burdock GA, Christian MS, Bitler CM, Crea R. 2006. Safety assessment of aqueous olive pulp extract as an antioxidant ao antimicrobial agent in foods. Food Chem. Toxicol. 44, 903-915.

Tajkarimi MM, Ibrahim SA, Cliver DO. 2010. Antimicrobial herb and spice compounds in food. Review Article. Food Control, 21, 1199-1218.

Tapiero H, Townsend DM, Tew KD. 2004. Organosulfur compounds from alliaceae in the prevention of human pathologies. Biomed. Pharmacother. 58, 183-193.

Thomson M, Al-Qattan KK, Bordia T, Ali M. 2006. Including garlic in the diet may help lower blood glucose, cholesterol, and triglycerides. J. Nutr. 136, 800S-802S.

USDA. 2007. Oxygen radical absorbance capacity (ORAC) of selected foods.

Wittig E. 2001. Evaluación Sensorial. Una metodología actual para tecnología de alimentos. Edición Digital reproducida con autorización del autor. En: [http:// mazinger.sisib.uchile.cl/repositorio/lb/ciencias_ quimicas_y_farmaceuticas/wittinge01/index.html].

Yin M, Cheng W. 2003. Antioxidant and antimicrobial effects of four garlic-derived organosulfur compounds in ground beef. Meat Sci. 63, 23-28.

Recibido: 4/10/10 Aceptado: $25 / 1 / 11$ 